\title{
Local Coherent-State approximation to system-bath quantum dynamics
}

\author{
R. Martinazzo* \\ Dept. of Physical Chemistry and Electrochemistry, \\ University of Milan, Golgi 19, 20113, Milan, Italy \\ I. Burghardt \\ Dept. de Chemie, Ecole Normal Superieure, \\ 24 rue Lhomond, f-75231 Paris cedex 05, France \\ F. Martelli \\ Dept. de Chemie, Ecole Normal Superieure, \\ 24 rue Lhomond, f-75231 Paris cedex 05, \\ France (on leave from Dept. of Physical Chemistry \\ and Electrochemistry, University of Milan)

\section{Nest} \\ Institute für Chemie, Universität Potsdam, \\ Karl-Liebknecht-Strasse 24-25, 14476 Potsdam, Germany
}

\section{INTRODUCTION}

System-bath dynamical problems arise naturally in chemical physics, e.g. when studying reactions in condensed phase or gas-surface processes. They represent one of the most challenging issues in current rate theories, especially when the need of a quantum description arises, as in the case of inherently quantum systems (e.g. hydrogen atom transfer in biologically relevant environments), and/or of low temperature media (e.g. the cold surfaces of the interstellar dust grains).

Our way to tackle this kind of problems is a 'brute-force' approach in which one follows the energy-conserving dynamics of a system coupled to a finite-size bath, and observes the dissipative dynamics (of the system) for times less than the Poincaré recurrence time. Such a (unitary) description of the total system + bath has been recently become a possible alternative [1-3] to open-system quantum dynamical approaches $[4,5]$ thanks to recent advances in quantum sim-

\footnotetext{
*Electronic address: rocco.martinazzo@unimi.it
} 
ulations of large systems. In this context, the main issue is the size of the bath (or, more properly, the average frequency spacing) which sets the limiting time scale for observing dissipative behaviour: in typical situations, a few ps process requires thousands of degrees of freedom, and this is beyond current computational possibilities, even for the Multi-Configuration Time-Dependent Hartree (MCTDH) method [6, 7], the most efficient, exact quantum dynamical method currently available. In order to make progress in this direction, some of the present authors have recently introduced a set of approximations, collectively named as Local Coherent-State approximation (LCSA) [8], to the unitary dynamics of a typical system-bath problem. The approximations are mainly in the bath description, whose dynamics is usually of no direct relevance, in the same spirit as in the reduced density operator approach. The resulting 'LCSA' approach turns out to be a selected-configuration MCTDH variant which closely resembles the so-called Gaussian-MCTDH [9-11] method, see below.

\section{THEORY}

We started by considering a typical system-bath hamiltonian which had been used for a long time to model dissipation (e.g. see Ref. 12-19), namely

$$
H=H^{s y s}+\sum_{k=1}^{F} \hbar \omega_{k}\left(a_{k}^{\dagger} a_{k}+\frac{1}{2}\right)-\sum_{k=1}^{F}\left(\lambda_{k}^{\dagger} a_{k}+\lambda_{k} a_{k}^{\dagger}\right)
$$

where $H^{\text {sys }}$ is the subsystem Hamiltonian, the second term on the r.h.s. is the Hamiltonian describing a harmonic bath and the third term represents the system-bath coupling, which was assumed to be linear in the bath coordinates but arbitrary in the subsystem coordinates; $a_{k}^{\dagger}, a_{k}$ are the usual harmonic oscillator (HO) raising/lowering operators. We can generalize it by writing

$$
H=H^{s y s}+H^{e n v}\left(. . q_{k} p_{k} . . ; \mathbf{x}\right)
$$

where $H^{e n v}$ is an 'environment' Hamiltonian (now comprising the coupling with the system) which is supposed to be local in system coordinates $\mathbf{x}$ and approximately harmonic in the bath degrees of freedom $\left(. . q_{k}, p_{k} ..\right)$. In this way one can include anharmonic bath oscillators, coupling between bath modes, etc... Our approximations come from the following observations concerning the Hamiltonian of Eq. (2): (i) coupling to the bath is local in subsystem coordinates, and (ii) the bath is approximately harmonic. Then, focusing on a wavefunction description appropriate for the $T=0 \mathrm{~K}$ case, point (i) suggests the use of subsystem Discrete-Variable-Representation (DVR) states in expanding the wavefunction for the total system, i.e.

$$
|\Psi\rangle=\sum_{\alpha} C_{\alpha}\left|\xi_{\alpha}\right\rangle\left|\Phi_{\alpha}\right\rangle
$$


where $\left\{\left|\xi_{\alpha}\right\rangle\right\}$ is a DVR set for the subsystem coordinates, and $\left|\Phi_{\alpha}\right\rangle$ are the resulting local bath states, one for each grid point $\alpha$ used to cover the relevant subsystem configuration space. In addition, point (ii) suggests that a product of $\mathrm{HO}$ coherent states (CSs) could be appropriate to describe the resulting local bath states, i.e.

$$
\left|\Phi_{\alpha}\right\rangle=\left|z_{\alpha}^{1}\right\rangle\left|z_{\alpha}^{2}\right\rangle . .\left|z_{\alpha}^{F}\right\rangle:=\left|Z_{\alpha}\right\rangle
$$

The result is that the bath dynamics is described by a set of coupled, pseudoclassical trajectories $z_{\alpha}^{k}=z_{\alpha}^{k}(t)$, one for each bath degree of freedom $k$ and system grid point $\alpha$. The system dynamics is contained in the time evolution of the amplitude coefficients $C_{\alpha}$, which make an important part of the system reduced density matrix $\left(\rho_{\alpha \beta}=C_{\alpha}^{*} C_{\beta}\left\langle Z_{\beta} \mid Z_{\alpha}\right\rangle\right.$ in the underlying DVR).

At finite temperature $T>0 \mathrm{~K}$ an analogous approach is possible as long as the initial system + bath density operator $\rho^{s b}$ can be written in the form

$$
\rho^{s b}=\sum_{i} p_{i}\left|\Psi_{i}^{L C S A}\right\rangle\left\langle\Psi_{i}^{L C S A}\right|
$$

where each $\left|\Psi_{i}^{L C S A}\right\rangle$ is a vector of LCSA form (eq. 3), which then undergoes the same energy-conserving time-evolution of the $T=0 \mathrm{~K}$ case. This is the case, for example, when the initial density operator $\rho^{s b}(0)$ can be factorized as $\rho^{s b}(0)=\rho^{s y s}(0) \otimes \rho_{\beta}^{b a t h}(0)$ and $\rho_{\beta}^{\text {bath }}(0)$ is a thermal density operator of a harmonic bath at temperature $k_{B} T=\beta^{-1}$. Indeed, using the CS representation of such thermal density operator, a Monte Carlo sampling of the resulting (bath) phase-space integral easily provides the set of 'realizations' of eq.4.

\section{Equations of motion}

Equations of motion can be derived with the help of the Dirac-Frenkel variational principle, using $C_{\alpha}$ and $z_{\alpha}^{k}$ as dynamical variables. When using conventional phase factors for the CSs (what we can call the "standard gauge") they take the following form.

The "system equation" is a kind of Schrödinger-Langevin equation

$$
i \hbar \dot{C}_{\alpha}=\sum_{\beta} H_{\alpha \beta}^{\text {damp }} C_{\beta}+v_{\alpha}^{\text {eff }} C_{\alpha}
$$

in which the elements of the system DVR hamiltonian are damped by the overlap between bath states, $H_{\alpha \beta}^{\text {damp }}=H_{\alpha \beta}^{\text {sys }}\left\langle Z_{\alpha} \mid Z_{\beta}\right\rangle$. The local, effective potential, $v^{e f f}=v^{l m f}+v^{\text {gauge }}$, contains a 'local mean-field' potential

$$
v_{\alpha}^{l m f}=\left\langle Z_{\alpha}\left|H^{e n v}\left(\mathbf{x}_{\alpha}\right)\right| Z_{\alpha}\right\rangle=H_{o r d}^{e n v}\left(. . z_{\alpha}^{k *}, z_{\alpha}^{k}, . . ; \mathbf{x}_{\alpha}\right)
$$


(here $H_{\text {ord }}^{e n v}$ is the environment hamiltonian operator expressed in terms in $a_{k}^{\dagger}, a_{k}$ and normally ordered, i.e. with all $a_{k}^{\dagger}$ 's on the left of $a_{k}$ 's) and a 'gauge' potential

$$
v_{\alpha}^{\text {gauge }}=-i \hbar \sum_{k=1}^{F}\left\langle z_{\alpha}^{k} \mid \dot{z}_{\alpha}^{k}\right\rangle=\hbar \sum_{k=1}^{F} \operatorname{Im}\left(z_{\alpha}^{k *} \dot{z}_{\alpha}^{k}\right)
$$

which can be explicitly written down with the bath equations below.

The "bath equations" are pseudoclassical equations

$$
i \hbar C_{\alpha} \dot{z}_{\alpha}^{k}=\sum_{\beta} H_{\alpha \beta}^{\text {damp }}\left(z_{\beta}^{k}-z_{\alpha}^{k}\right) C_{\beta}+C_{\alpha} \frac{\partial H_{\text {ord }}^{e n v}}{\partial a_{k}^{\dagger}}\left(. . z_{\alpha}^{k} z_{\alpha}^{k *}, . . ; \mathbf{x}_{\alpha}\right)
$$

containing a 'classical, local force'

$$
\dot{z}_{\alpha, \text { class }}^{k}=-\frac{i}{\hbar} \frac{\partial H_{o r d}^{e n v}}{\partial a_{k}^{\dagger}}\left(. . z_{\alpha}^{k} z_{\alpha}^{k *}, . . ; \mathbf{x}_{\alpha}\right)
$$

and a 'quantum' one

$$
\dot{z}_{\alpha, q u a n t}^{k}=-\frac{i}{\hbar C_{\alpha}} \sum_{\beta} H_{\alpha \beta}^{\text {damp }}\left(z_{\beta}^{k}-z_{\alpha}^{k}\right) C_{\beta}
$$

coupling CSs of the same degree of freedom at different grid points. The latter is essential for a quantum, though approximate, description of the bath dynamics. The above gauge potential can then be easily written down with the help of the matrix

$$
\Gamma_{\alpha \beta}=\sum_{k=1}^{F} z_{\alpha}^{k *} z_{\beta}^{k}
$$

which is also useful to evaluate the CSs overlaps, $\left\langle Z_{\alpha} \mid Z_{\beta}\right\rangle=\exp \left(\Gamma_{\alpha \beta}-\Gamma_{\alpha \alpha} / 2-\right.$ $\left.\Gamma_{\beta \beta} / 2\right)$. For a derivation of the equations see Ref. 8 , and notice that, in general, $\left[a, f_{\text {ord }}\left(a^{\dagger}, a\right)\right]=\partial f_{\text {ord }}\left(a^{\dagger}, a\right) / \partial a^{\dagger}$.

\section{General properties}

The above equations have some interesting properties. Some of them arise from the use of the Dirac-Frenkel variational principle. Norm and energy conservation are guaranteed with our ansatz (since it is also an allowed variation) and can be used in practice to check the quality of the propagation. More generally, the solutions of the LCSA equations define a hamiltonian flow in the space of the parameters, i.e. the equations have a symplectic structure that can be 
used to set up a robust propagation scheme. We'll come back to this point in Section IV.

Other properties arise from the LCSA ansatz itself. The bath dynamics is reduced to a set of trajectories, whose number scales linearly with the bath dimensions. This means that the method itself has a power-low scaling with such dimensions, the exponent of this scaling depending on the interaction between bath modes. For bath modes coupled to the system only (e.g. with the hamiltonian of Eq. 1) linear scaling has been observed and model simulations with tens of thousands of bath degrees of freedom have been performed on modest computers [8]. This good scaling property is in common with mixed quantum-classical methods, which however fail to correctly represent the system-bath correlations. In LCSA a number of trajectories is used for each bath degree of freedom, and they are coupled to each other by the building up of the (quantum) correlation. Note also that in our case the system 'wavefunction' (the amplitude coefficients) enters linearly in the bath equations, and therefore phase factors do play a role in the classical dynamics, apart from an overall phase factor.

Coupled trajectories arise in a number of closely related approaches, namely the Coupled-Coherent-State method of Shalashilin and Child [20-23] and the Gaussian-MCTDH method of Burghardt, Worth and coworkers [9-11]. The latter, in particular, is strongly connected with LCSA. The two approaches share a conventional, 'exact' description of some interesting degrees of freedom and an approximate, CS description of other, less interesting degrees of freedom. Indeed, as a matter of fact, the G-MCTDH equations [9] reduce to LCSA ones under suitable constraints (e.g. see Appendix B of Ref. 8). The main difference between the two is that in LCSA all the configurations are orthogonal to each other, as a consequence of the presence of a different DVR state in each of them. This leads to considerable simplifications in the resulting equations (see above, in particular, the bath equations), at the price of a reduced accuracy.

Finally, one interesting property about the pseudo-classical description of the bath degrees of freedom is that it suits well to induce dissipative dynamics into the total system. This can be accomplished by adding a suitably designed friction coefficient $\eta$ to the bath equations, mimicking the presence of a secondary (infinite, memory-less) bath. More formally, it can be shown that applying the LCSA approximation to a system + bath + secondary bath configuration, a classical approximation to the secondary bath dynamics, and standard assumptions (Ohmic bath in the continuum limit) a friction coefficient appears in the LCSA equations for the system+bath degrees of freedom[28] (e.g. see Appendix A of Ref. 8). This possibility has been exploited, especially in conjunction with the need of removing numerical instabilities of the method without altering the system dynamics. We call such a modified version of our approach the 'damped' LCSA variant, for obvious reasons. 


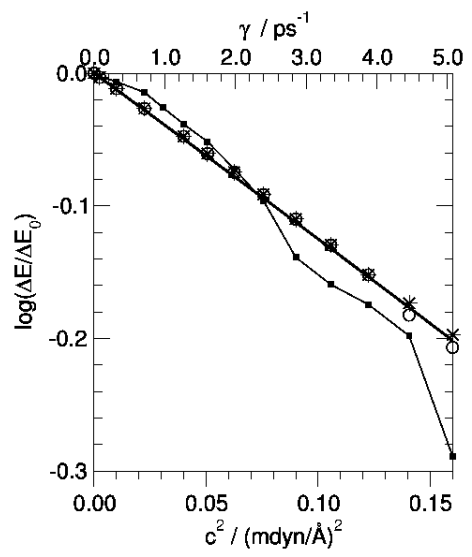

Figure 1: A double-well system coupled to a 1D harmonic bath oscillator: tunneling splitting as a function of the coupling strength. Bold lines for exact results, square symbols for standard LCSA. Very good results can be obtained by employing the damped version of LCSA (stars), even when the fictitious damping is turned on for the first $0.1 \mathrm{ps}$ only (empty circles). Here, damping helps in removing an 'initialization' problem, see Ref. 8 for details.

\section{APPLICATIONS}

The method has been applied to a number of model problems, ranging from tunneling to vibrational relaxation and sticking dynamics, and the results have been compared with those of exact, Multi-Configuration-Time-DependentHartree calculations in systems with up to 80 bath oscillators. A detailed account of such a comparison is given in Ref. 8. Here we merely summarize the main results.

When the method is applied to problems with small dimensional baths (e.g. an oscillator coupled to few $\mathrm{HO}$ bath oscillators) it performs rather well, and the results are in close agreement with exact ones. This is particularly true for 1D bath problems, in which LCSA is free of the (local) Hartree approximation, thereby suggesting that constraining the local bath states to be CSs is a reasonably good approximation. The correct treatment of system-bath correlation is evident in the test problem of a double well system coupled bilinearly to a harmonic oscillator[24]. Fig.1 shows, for example, the behavior of the tunneling splitting for different values of the coupling strength $c$, compared to exact results. Notice for comparison that a time-dependent self-consistent field approach to this problem would badly fail in describing the tunneling dynamics at all but very small coupling strengths [24]. 

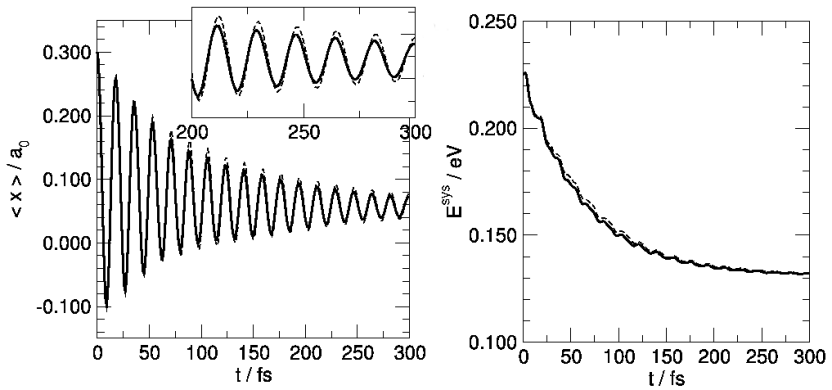

Figure 2: Average position (left) and system energy (right) for a 50D vibrational relaxation dynamics with relaxation time $\gamma^{-1}=50 \mathrm{fs}$. Full lines for converged MCTDH results, and dashed lines for (damped) LCSA calculations. The latter take $\sim 1$ min on a standard desktop computer.

Application of the method to large-dimensional baths show the main limitation of the present approach. The vibrational relaxation dynamics of an anharmonic oscillator coupled to a 50D bath, as described by standard LCSA, is in only qualitative agreement with converged MCDTH calculations. A detail analysis suggests that this is more probably due to numerical difficulties than to real limitations of the LCSA approach. For example, time-reversal invariance is lost after a few tens of fs, discrepancies increase in weaker-coupled systems (long time dynamics), and, in general, long-time results show some dependence on numerical parameters (e.g. the grid spacing defining the Colbert-Miller DVR used in the calculations). This is a much more subtle problem that it seems at first sight; as an example, we notice that our integrator (a standard Runge-Kutta 4 -th order one) allows very good norm and energy conservation. Though some improvement has been recently found by employing better-suited DVR sets (in particular, the Potential-Optimized DVR set based on the eigenvectors of the system hamiltonian)[25], the working strategy at present is to use the damped version of LCSA. The fictitious friction coefficient $\eta$ of the previous Section can be optimized with a minimum effort without reference to any benchmark result, and turns out be essentially a bath property. The use of such a 'trick' allows one to get results in very good agreement with exact ones in an extremely cheap way, see Fig. 2. Likewise, one can 'stabilize' the bath dynamics in tunneling problems without affecting the system dynamics, as we have shown in Fig.1 and observed in other, large dimensional tunneling problems.

One interesting feature of the 'damped' version of LCSA is that the introduction of a friction coefficient $\eta$, by inducing an overall dissipative dynamics, completely removes the bath recurrence problem. This is shown in Fig.3 where different discretizations of the same bath (i.e. which preserve the spectral den- 

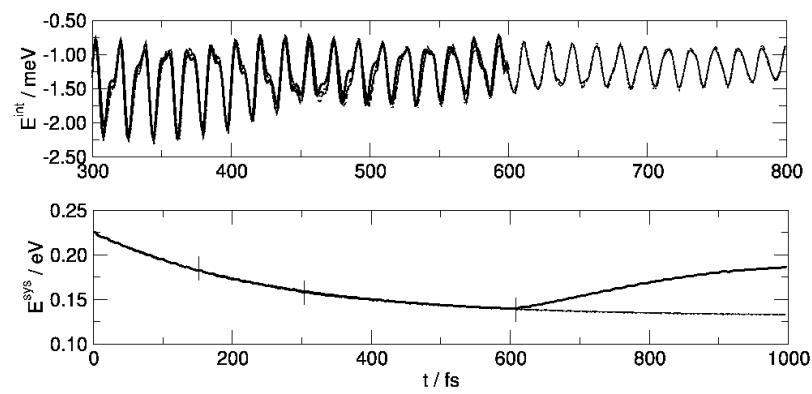

Figure 3: A vibrational relaxation dynamics showing the removal of the recurrence problem when the damped version of LCSA is used. Bold lines for MCTDH calculations with 80 bath modes, solid, dashed and dotted lines for LCSA calculations with 20,40, and 80 modes. Vertical bars mark the corresponding 'nominal' recurrence times. Top: average interaction energy. Bottom: average system energy.

sity $J(\omega)$ ) give the same (essentially exact) results for times much larger than their corresponding recurrence times.

Thus, the introduction of a simple damping coefficient in the pseudoclassical bath equations seems to solve the numerical instability problems, and introduce nice features in the method. This, however, cannot be the final solution. This is clear, for example, when considering a sticking dynamics (i.e. by using the bath to model a surface). In this case, the standard version of LCSA works reasonably well, and correctly reproduces the energy transfer. However, when looking at detailed quantities such as the sticking probability it is clear that dynamics does not proceed 'smoothly', see Fig.4. The bad break here is that when we introduce the damping coefficient we do stabilize the bath dynamics but at the price of having no more sticking (!). This is clearly due to the important role that low-frequency bath oscillators play in the dynamics: the use of a frequency dependent damping coefficient can improve the situation, but this seems to be a too ad hoc procedure. For this reason, we have been currently looking for much more robust propagation algorithms than the ones we have adopted so far. This motivated a deeper understanding of the properties of the working equations, as it is sketched in the following Section.

\section{HAMILTONIAN FLOWS}

The results obtained so far were based on 'brute-force' solution of the firstorder LCSA equations of motion. However, a good propagator should take care of preserving their (non-evident) features, namely their symplectic structure. Indeed, it has been known for some time[26, 27] that any variational quantum 

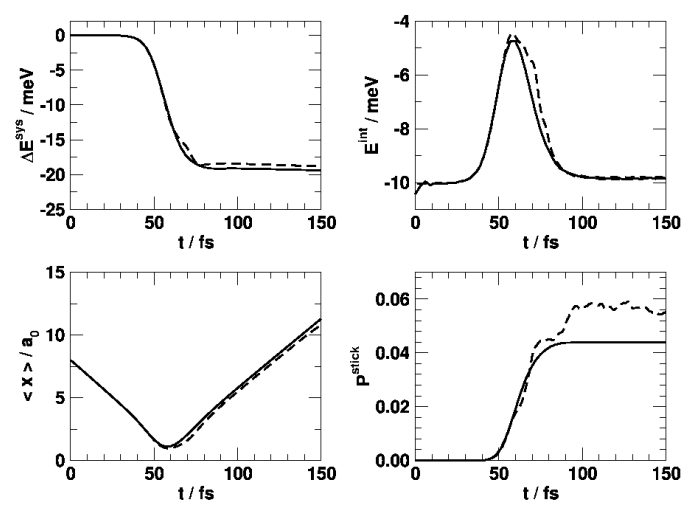

Figure 4: A model sticking dynamical problem, describing a $\mathrm{H}$ atom colliding with a 50D-mode surface at a collision energy of $200 \mathrm{meV}$. Average system energy loss $\left(\Delta E^{s y s}\right)$, interaction energy $\left(E^{i n t}\right)$, position $(<x>)$ and sticking probability $\left(P^{\text {stick }}\right)$ as a function of time. Solid lines for MCTDH results, dashed lines for LCSA.

method (under quite mild regularity conditions) can be recast in the form of a symplectomorphism on a symplectic manifold. This means in practice that rather than using a brutal time discretization one can better approximate the short-time dynamics of the system by a composition of (simple) symplectic maps in order to keep trace of this extraordinary property. In the following we summarize the important points in this respect, leaving an ampler discussion to a forthcoming paper.

Let's first briefly introduce the concept of hamiltonian flows in generic symplectic manifolds. A symplectic manifold is a differentiable manifold equipped with a closed, non-degenerate 2-form $\omega$. In a coordinate system $x^{i}$ it can be written as $\omega=\sum_{i, j>i} \omega_{i j} d x^{i} d x^{j}$. Here $d x^{i}$ are the fundamental 1-forms, that is $d x^{i}(\mathbf{v})=v^{i}$ for any tangent vector $\mathbf{v}$ in a given point $\mathbf{x}$, and the product of differentials is the so-called 'wedge' product. Non-degeneracy means in practice that $\omega_{i j}$ is non-singular everywhere in the manifold[29], and this allows one to set up a one-to-one map between tangent and co-tangent vectors (1-forms). That is, for a given 1-form $\alpha=\sum \alpha_{i} d x^{i}$ there is an associated vector field $\mathbf{X}_{\alpha}$ such that $\alpha(\mathbf{v})=\omega\left(\mathbf{X}_{\alpha}, \mathbf{v}\right)$, and its flow, defined by the curves $\dot{x}^{i}=X_{\alpha}^{i}$. Then, given a smooth function $H$ (which can be called a hamiltonian) and its 1-form $d H$ the flow induced by its associated vector field $\mathbf{X}_{H}$ (what can be called a hamiltonian flow) conserves the function itself, $d H\left(\mathbf{X}_{H}\right)=\omega\left(\mathbf{X}_{H}, \mathbf{X}_{H}\right)=0$. Closedness ( $d \omega=0$ where $d$ is the 'exterior' derivative) means that these properties can be 'transported' along the manifold, and guarantees that the symplectic form $\omega$ itself is invariant under any hamiltonian flow (formally $\mathcal{L}_{X_{H}} \omega=0, \mathcal{L}_{Y}$ being 
the Lie derivative along the vector field Y. This forms the basis for Liouville's theorem).

In our context, it is worth noticing that a 'variational' method can be defined with the help of the time-dependent variational principle, namely $\delta S=0$. Here the action $S=\int_{t_{1}}^{t_{2}} d t L$ is given by the (real) Lagrangian[26, 27]

$$
L=\frac{i \hbar}{2} \frac{\langle\Psi \mid \dot{\Psi}\rangle-\langle\dot{\Psi} \mid \Psi\rangle}{\langle\Psi \mid \Psi\rangle}-\frac{\langle\Psi|H| \Psi\rangle}{\langle\Psi \mid \Psi\rangle}
$$

The time dependent variational principle gives back the time-dependent Schrödinger equation when $|\Psi\rangle$ is allowed to vary in the whole space, without constraints. It is implied by the Dirac-Frenkel one. The converse is also true when $i|\delta \Psi\rangle$ is an allowed variation for any possible variation $|\delta \Psi\rangle$.

Then, a general symplectic structure can emerge when (smoothly) introducing a set of variational parameters $\mathbf{x},|\Psi\rangle=|\Psi(\mathbf{x})\rangle$, forming a coordinate system in the manifold of the 'sample' space. This can be made up, for example, by the real and the imaginary parts of a set of $n$ complex parameters, such as our $\left\{. . C_{\alpha} . . z_{\alpha}^{k} ..\right\}$. In terms of this parametrization,

$$
L=\sum_{i=1}^{n} \dot{x}^{i} Z_{i}(\mathbf{x})-\mathcal{H}(\mathbf{x})
$$

where

$$
Z_{i}=\frac{i \hbar}{2\langle\Psi \mid \Psi\rangle}\left(\left\langle\Psi \mid \frac{\partial \Psi}{\partial x^{i}}\right\rangle-\left\langle\frac{\partial \Psi}{\partial x^{i}} \mid \Psi\right\rangle\right)
$$

are the components of a 1-form $\alpha=-\sum Z_{i} d x^{i}$, and $\mathcal{H}(\mathbf{x})=$ $\langle\Psi(\mathbf{x})|H| \Psi(\mathbf{x})\rangle /\langle\Psi(\mathbf{x}) \mid \Psi(\mathbf{x})\rangle$. A closed 2-form can then be defined as $\omega=d \alpha$, and it is non-degenerate if $\omega_{i j}=\partial Z_{i} / \partial x^{j}-\partial Z_{j} / \partial x^{i}$ is non-singular. In this case, the 2 -form $\omega=\sum \omega_{i j} d x^{i} d x^{j}$ provides the symplectic structure we need.

Indeed, the equations of motion defined by the time-dependent variational principle follow by the above Lagrangian in the form

$$
\sum \dot{x}^{i} \omega_{i j}=\frac{\partial \mathcal{H}}{\partial x^{j}}
$$

or equivalently, for a generic tangent vector $\mathbf{v}$,

$$
\sum \dot{x}^{i} \omega_{i j} v^{j}=\omega(\dot{\mathbf{x}}, \mathbf{v})=\sum \frac{\partial \mathcal{H}}{\partial x^{j}} d x^{j}(\mathbf{v})=d \mathcal{H}(\mathbf{v})
$$

It follows that if $\omega$ is a symplectic form, the 'variational flow' is the hamiltonian flow of the hamiltonian $\mathcal{H}(\mathbf{x})$, i.e. $\dot{\mathbf{x}}=\mathbf{X}_{\mathcal{H}}$. The variational equations can also be written with the help of Poisson brackets

$$
\dot{x}^{i}=\left\{H, x^{i}\right\}
$$


which are defined by $\{f, g\}=\omega\left(\mathbf{X}_{g}, \mathbf{X}_{f}\right)$ for any two smooth functions $f$ and $g$. In a coordinate system they are given explicitly by

$$
\{f, g\}=\sum \frac{\partial f}{\partial x^{i}} \frac{\partial g}{\partial x^{j}} \xi^{i j}
$$

where $\xi^{i j}$ is the matrix inverse of $\omega_{i j}$.

A detailed analysis of the LCSA case [25] reveals that the relevant form $\omega$ is indeed non-degenerate in the whole parameter space where none of the amplitude coefficients $C_{\alpha}$ is zero. This is consistent with the ansatz of Eq. 3 since when one the $C_{\alpha}$ vanishes the associated CSs $z_{\alpha}^{k}$ are irrelevant for the dynamics.

\section{SUMMARY}

In this paper an account has been given of the current status of what we have called the Local Coherent-State Approximation, and its related quantum dynamical approach to system-bath problems. An attempt has been made to show its merits and present limitations. On the one hand, we have shown that the method can be very accurate and extremely cheap, very nice features for possible realistic applications. On the other hand, we have also shown that numerical stability problems have been preventing straightforward application of the approach. In an attempt to overcome these difficulties, we have also sketched our ongoing search of a robust propagation scheme.

Acknowledgements Gian Franco Tantardini and Peter Saalfrank are greatly acknowledged for their continuous and active involvement in the project.

[1] M. Nest and H.-D. Meyer, J. Chem. Phys. 119, 24 (2003).

[2] I. Burghardt, M. Nest, and G. A. Worth, J. Chem. Phys. 119, 5364 (2003).

[3] H. Wang and M. Thoss, J. Chem. Phys. 119, 1289 (2003).

[4] H.-P. Breuer and F. Petruccione, The theory of open quantum systems (Oxford, Oxford, 2002).

[5] U. Weiss, Quantum dissipative systems, vol. 13 of Series in Condensed Matter Physics (World Scientific, 2008).

[6] M. H. Beck, A. Jackle, G. A. Worth, and H.-D. Meyer, Phys. Rep. 324, 1 (2000).

[7] H.-D. Meyer and G. Worth, Theor. Chem. Acc. 109, 251 (2003).

[8] R. Martinazzo, M. Nest, P. Saalfrank, and G. F. Tantardini, J. Chem. Phys. 125, $194102(2006)$.

[9] I. Burghardt, H.-D. Meyer, and L. S. Cederbaum, J. Chem. Phys. 111, 2927 (1999).

[10] G. A. Worth and I. Burghardt, Chem. Phys. Lett. 502, 368 (2003).

[11] G. A. Worth, M. A. Robb, and I. Burghardt, Faraday Discuss. 127, 307 (2004).

[12] R. J. Rubin, J. Math. Phys. 1, 309 (1960). 
[13] J. R. Senitzky, Phys. Rev. 119, 670 (1960).

[14] R. Zwanzig, J. Chem. Phys 32, 1173 (1960).

[15] R. Zwanzig, J. Stat. Phys. 9, 215 (1973).

[16] S. A. Adelman and J. D. Doll, J. Chem. Phys. 64, 2375 (1976).

[17] J. C. Tully, J. Chem. Phys. 73, 6333 (1980).

[18] A. O. Caldeira and A. J. Leggett, Ann. Phys.(N.Y.) 149, 374 (1983).

[19] E. Cortes, B. J. West, and K. Lindeberg, J. Chem. Phys. 82, 2708 (1985).

[20] D. V. Shalashilin and M. S. Child, J. Chem. Phys. 119, 1961 (2003).

[21] D. V. Shalashilin and M. S. Child, J. Chem. Phys. 121, 2563 (2004).

[22] D. V. Shalashilin and M. S. Child, J. Chem. Phys. 122, 224108 (2005).

[23] D. V. Shalashilin and M. S. Child, J. Chem. Phys. 122, 224109 (2005).

[24] N. Makri and W. H. Miller, J. Chem. Phys. 87, 5781 (1987).

[25] R. Martinazzo (unpublished).

[26] A. K. Kerman and S. E. Koonin, Annals of Physics 100, 332 (1976).

[27] P. Kramer and M. Saraceno, Geometry of the time-dependent variational principle in quantum mechanics, vol. 140 of Lecture Notes in Physics (Springer-Verlag, Berlin Heidelberg New York, 1981).

[28] The same applies to finite temperature cases where, as expected, both a friction and a fluctuating term appear in the LCSA equations of each realization of eq.4.

[29] This condition restricts the analysis to even dimensional manifolds. 